\title{
Rooibos Tea and Health: A Systematic Review of the Evidence from the Last Two Decades
}

\author{
Timothy Bond $\mathrm{J}^{1}$, and Emma Derbyshire $\mathrm{J}^{2 *}$ \\ ${ }^{1}$ Tea Advisory Panel; Tea and Herbal Solutions, Bedford, United Kingdom \\ ${ }^{2}$ Nutritional Insight, Epsom, Surrey, United Kingdom
}

*Corresponding author: Emma Derbyshire J, Nutritional Insight, Epsom, Surrey, United Kingdom, E-mail: emma@nutritional-insight.co.uk

Received: 08 May, 2020 | Accepted: 29 May, 2020 | Published: 05 Jun, 2020

Citation: Bond TJ, Derbyshire EJ (2020) Rooibos Tea and Health: A Systematic Review of the Evidence from the Last Two Decades. Nutr Food Technol Open Access 6(1): dx.doi.org/10.16966/2470-6086.166

Copyright: (c) 2020 Bond TJ, et al. This is an open-access article distributed under the terms of the Creative Commons Attribution License, which permits unrestricted use, distribution, and reproduction in any medium, provided the original author and source are credited.

\begin{abstract}
An expanse of research has investigated the effects of black and green teas in relation to aspects of health. Rooibos tea, also known as Red bush is derived from the South African Cape fynbos plant, Aspalathus linearis, and is caffeine free, naturally sweet and abundant in polyphenols. Evidence related to the health aspects of drinking Rooibos tea is advancing, but does not appear to have been collated. Therefore, we aimed to examine the health effects of Rooibos tea through a systematic review of the literature. A PUBMED search was undertaken (2000 up to June 2020) for human and laboratory studies investigating the efficacy of Rooibos in relation to health. Seven human studies and 49 laboratory studies were identified. Overall Rooibos tea consumption seems to benefit the lipid and redox profiles of those at risk of cardiovascular disease. It also appears to possess other promising 'general' effects on glycaemic control, bone, liver, cognitive and respiratory health. Ongoing research using standardised interventions is now needed to help formulate congruent conclusions that are relevant to public health.
\end{abstract}

Keywords: Rooibos tea; Poly phenols; Health; Evidence-base

\section{Introduction}

The pattern of health and disease is changing-continued shifts in longevity mean that multimorbidity and 'disease clusters' are now on the rise [1]. It is well appreciated that lifestyle e.g. alterations in diet, physical activity and avoiding smoking can improve outcomes for medical conditions such as cardiovascular disease [2]. There is already an extensive body of evidence showing that drinking two to three cups of tea daily could be beneficial for health, including reduced risk of cardiac death, coronary artery disease, stroke, type 2 diabetes mellitus and total mortality [3]. Beneficial inter-relationships have also been observed for several cancers, cognitive, skeletal and maternal health [3]. Most of this research has focused on green, black and oolong tea [4].

"Rooibos" is Afrikaans for "red bush" [5]. It is prepared from unfermented and fermented plant material from the Cape fynbos plant, Aspalathus linearis [6,7]. In South Africa, the proportion of black tea drinkers has declined between 2011 and 2015, from 58.6\% to $51.5 \%$ whilst the percentage of Rooibos consumers has risen from $29.4 \%$ in 2011 to $30.9 \%$ in 2015.8 The demand for Rooibos tea is also extending further afield with South Africa exporting Rooibos tea to more than 30 countries [8].

Such shifts in consumption habits are being attributed to rising awareness and interest in the health properties of Rooibos tea [8].
The health properties of rooibos tea have been ascribed to it being caffeine free and its abundant phenolic composition $[9,10]$. Rooibos tea has a divergent polyphenol profile and is a rare dietary source of dihydrochalcones-aspalathin and nothofagin which possess potent antioxidant actions [11-13]. Aspalathin and nothofagin are the main flavonoids in Rooibos tea and have a particularly strong antioxidative activity, though aspalathin tends to be lower in fermented than unfermented foods $[10,12,14]$. This is relevant to health as the antioxidants present in rooibos may help to protect against oxidative stress which is known to induce inflammation and other health conditions $[10,15]$.

Rooibos tea is naturally slightly sweet with caramel, floral, honey and woody undertones [16]. It has been used both as a tisane, as well as consumed traditionally for medicinal purposes and has been popular in South Africa for generations [5,11]. Traditionally, rooibos has been used for its medicinal properties in South Africa to help alleviate allergies, asthma, dermatological conditions and infantile colic [17]. Both the leaves and fine stems can be used as herbal tea, predominantly in the traditional 'fermented' (oxidised/aerated) redbrown form but also in its 'unfermented' (unoxidized/aerated) green form [18]. Rooibos tea can also be extracted and dried, spray-dried/ freeze-dried to form powdered rooibos tea extract (RTE) which is also abundant in polyphenols [5]. Traditionally fermented beverages such as Kombucha have been produced effectively using rooibos leaves [19]. 
Increasingly, rooibos has been studied in human populations, mainly for its antioxidant and cardio protective properties [14,20,21]. Alongside this, over the years a growing body of laboratory and mechanistic studies have investigated how rooibos tea could impact on health. Given the gaining popularity of rooibos tea, the current publication collates evidence from human and laboratory studies, published over the last two decades. Such a review does not appear to have been undertaken previously. The present review focuses on rooibos tea which has been gaining popularity both in its native province the Western Cape of South Africa and worldwide in recent years [8].

\section{Methods}

The National Centre for Biotechnology Information (NCBI) search engine (PubMed) was used to extract relevant publications. Two search phases were undertaken. In Phase 1 English-language human studies published between January 2000 (month start) and June 2020 (month start) were screened. Publications were included if they used Rooibos tea or RTE and studied a named health outcome. Studies were excluded if they were conducted with children due to potential ethical issues, focused on chemical profiling, used a multi-intervention or did not specify a distinctive health outcome. External health conditions such as skin healing were also excluded.

The search terms "Rooibos", "Red bush" or "Aspalathus linearis" were used. ED and TB identified the scientific publications. In Phase 1 the database search was restricted to human studies. In Phase 2 the search was restricted to laboratory studies the same search terms were applied. Data extracted from each human study included: (1) Study (author, year, location and reference number), (2) Subjects (age, gender, number), (3) Study design (type), (4) Tea intervention (type) (5) Intervention type (dosage) and (6) Main findings. Data extracted from each laboratory/mechanistic study included: (1) Study (author, year and reference number), (2) Study Design, (3) Intervention (type), (4) Main outcome and (5) Main findings.

\section{Results}

In Phase 1 using the applied search terms 47 human studies were identified. Of these, three were review papers, three had methods that were unclear or not fully reported, five were laboratory studies and 29 irrelevant as they did not investigate health outcomes. Subsequently, after these exclusions seven human studies were included in the final review. Of the human studies three were conducted in South Africa [21-23], one in the USA [24], one in Italy [14], one in Sweden [25] and one in Germany [20].

In Phase 291 laboratory studies were first identified. Two of these were excluded due to them being multi-interventions, two focused on external (skin) conditions, four were review papers, seven had methods that were unclear or not fully reported and 27 were irrelevant as they did not measure health outcomes. This resulted in 49 laboratory studies being included in the main paper. The algorithm of qualifying papers is shown in figure 1.

\section{Human Studies}

Seven human studies were identified using Rooibos tea preparations (Table 1) [14,20-25]. Sample sizes ranged from eight to 40 subjects. Interventions also varied between studies. Most studies used Rooibos infusions that had been seeped for approximately 10 minutes in hot water infusions $[20,23,25]$. One study provided Rooibos tea (two 125 $\mathrm{ml}$ cups each made with two tea bags, low mineral content water and a brewing time of $5 \mathrm{~min}$ at $90^{\circ} \mathrm{C}$ ) for 30 days [23]. Other research provided six cups of fermented, traditional Rooibos daily (one tea bag in $200 \mathrm{ml}$ with an infusion time of 5 minutes) for 6 weeks [21].

Three studies reported positive findings in relation to aspects of health $[21,22,25]$. One study used ex vivo samples from atopic adults showing that extracts prepared from both fermented and unfermented Rooibos inhibited basophil activation, an effect that was stronger using the extract of fermented Rooibos [22]. These findings are aligned with that from earlier laboratory models [26] and indicate that Rooibos appears to possess anti-allergic effects by inhibiting antigen- and calcium ionophore-stimulated degranulation. One of the largest trials conducted on 40 participants observed significant increases in plasma total polyphenol levels, reductions in markers of lipid peroxidation, improvements in lipid profiles (low-density lipoprotein declined and high-density lipoprotein increased) and redox status [21]. Effects were observed after drinking six cups of fermented (traditional) Rooibos tea daily for 6 weeks [21]. Another trial looking into underpinning mechanisms showed that freshly prepared Rooibos tea (made with $10 \mathrm{~g}$ tea in $400 \mathrm{ml}$ boiled water for $10 \mathrm{~min}$ using a tea filter) significantly inhibited angiotensin-converting enzyme (ACE) activity, 30 and 60 minutes after ingestion, indicating possible cardiovascular effects via the inhibition of ACE activity [25].

Two human studies focused on aspects of metabolite absorption and bioavailability [14,20]. In a human crossover study where $500 \mathrm{ml}$ unfermented Rooibos tea was ingested aspalathin (a dihydrochalcone C-glucoside) was found to be particularly bioavailable [20]. An earlier bioavailability trial comprised of 10 adults drinking a similar quantity of Rooibos tea also showed that most metabolites were absorbed either via the small or large intestine [14]. In two trials involving Rooibos tea ingestion, one did not show any significant effects on renal stone formation [23] and the other showed that rooibos tea and plain water similarly rehydrated 23 athletes [24].

\section{Laboratory Studies}

A wealth of research has studied the health effects and potential mechanisms of Rooibos tea and its associated flavonoids. Forty-nine laboratory/mechanistic studies were identified and published over the last two decades. Of these, eleven focused on aspects of oxidative stress and antioxidant activity [10,12,27-35]. Four studies observed improvements in dimensions of sperm function [36-39]. This included enhanced sperm velocity, vitality, acrosome structure and membrane integrity [36 38]. These effects were attributed to high levels of antioxidants found in Rooibos, sequestering reactive oxygen species and lipid peroxidation $[38,39]$.

Other research has focused on aspects of metabolic health. Some work has found associations between unfermented/green Rooibos extract and improvements in fasting blood glucose levels using type 2 diabetic mice [40]. Similarly, In vitro work showed that Rooibos extract high in aspalathin had a sustained glucose lowering effect [41]. Other work indicated that aspalathin or nothofagin flavonoids inhibit glucose-mediated vascular hyperpermeability and inflammation [42]. A further seven studies showed that Rooibos could improve insulin resistance and have antidiabetic potential [43-49].

With regard to potential mechanisms aspalathin found in Rooibos stimulated glucose uptake in muscle tissues and insulin secretion from pancreatic beta-cells in a type 2 diabetes mouse model [48]. Work by Ulicna O, et al. (2006) found that the antioxidant compounds in Rooibos tea prevented oxidative stress concluding that it could be a suitable adjunctive therapy for diabetic vascular conditions [49]. One study using a cell model found that a fermented Rooibos infusion prepared at 'cup-of-tea' strength and the soluble matter of the infusion 


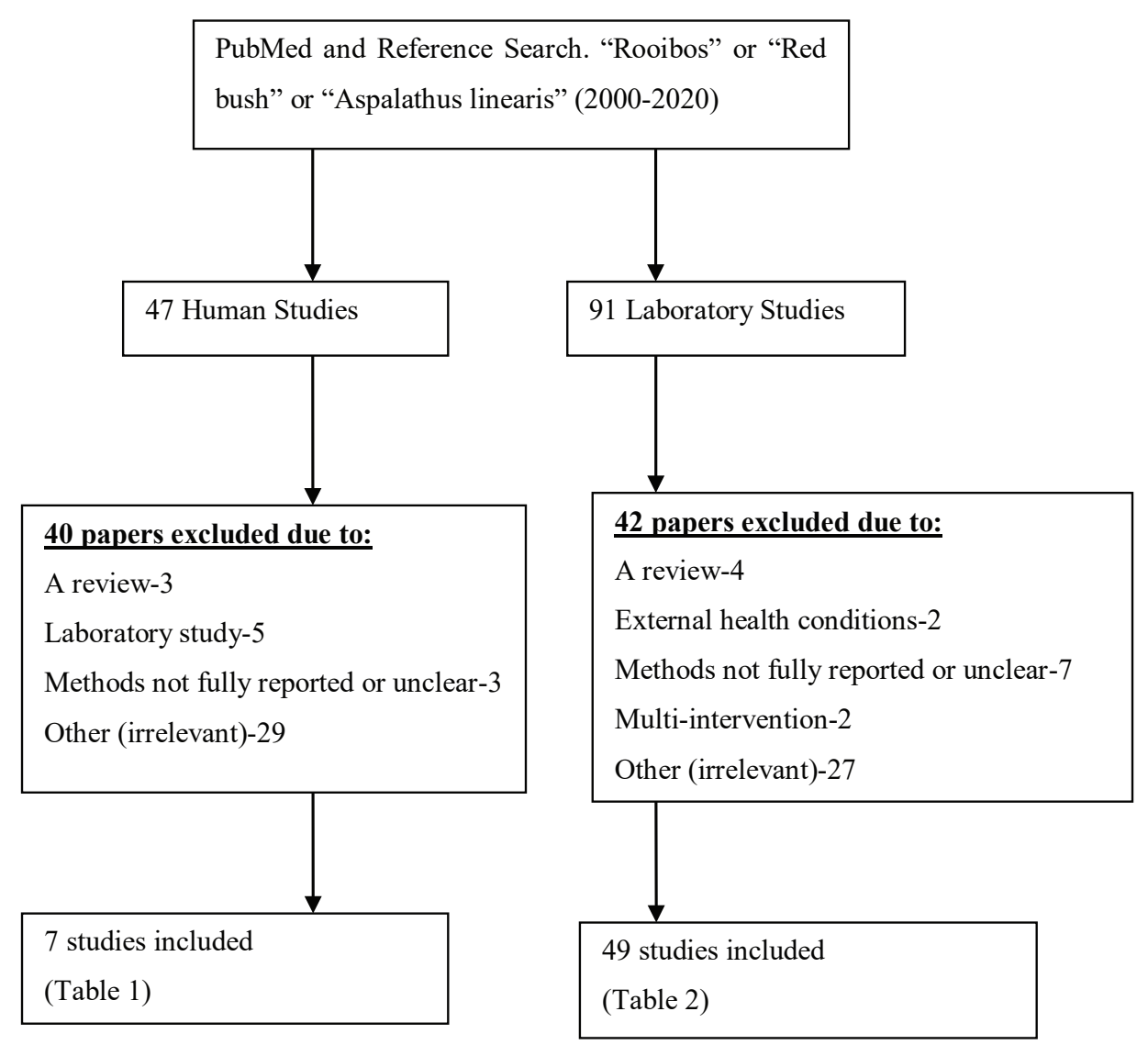

Figure 1: Algorithm for database search results.

inhibited adipogenesis, also implying a potential role in obesity prevention [50]. These findings imply a potential role in glycaemic regulation.

Other research suggests beneficial effects on aspects of bone health [51,52], hepatoprotection [31,43,44,53], allergic response [26] and immune function [54,55]. Rooibos tea has been found to improve osteoblast activity [52] while fermented Rooibos was found to inhibit osteoclasts and associated gene expression [51]. Some work has discovered that Rooibos tea could help to stabilise the liver from injury [12]. In another study Rooibos extract eased induced liver injury by suppressing oxidative stress and the formation of pro-inflammatory cytokines [31]. A laboratory model showed that Rooibos tea acted as a 'hepatoprotector' showing histological regression of liver cirrhosis and steatosis in an experimental model of liver cirrhosis [53].

Alongside these findings, other evidence from laboratory models suggests inter-relationships between Rooibos ingestion and improvements in spatial memory, [56] reduced brain oedema and neuronal apoptosis, [57] reductions in esophageal papilloma size, [58] antispasmodic effects, [59,60] bronchodilation, [60] and chemoprotection $[61,62]$.

\section{Discussion}

Overall there appears to be a growing body of evidence relating to Rooibos tea and various biological health outcomes. The largest body of evidence is currently derived from laboratory and mechanistic studies although human research is emerging. Research focusing on cardiovascular health looks particularly promising. The trial conducted by Marnewick JL, et al. (2011) [21] was well conducted showing that drinking six cups of fermented, traditional rooibos daily significantly improved the lipid profile and redox status which is relevant to adults at risk for developing cardiovascular disease. Elsewhere other review findings also conclude that Rooibos appears to have preventative and complementary therapeutic benefits in the context of cardiovascular disease [63].

The antioxidant properties of Rooibos tea and its ability to sequester oxidative stress are also prominent in the research $[27,34,49,64]$. The polyphenols aspalathin (present at $>5 \mathrm{mg} / \mathrm{l}$ ) and nothofagin (present at $<1 \mathrm{mg} / \mathrm{L}$ ) found in Rooibos tea have been attributed to some of its health benefits $[65,66]$. Antioxidants such as these suppress oxidative stress in the body which has been implicated in the pathophysiology of certain diseases including Alzheimer's disease [67]. Several laboratory studies concentrated on aspects of sperm function [36-39]. Fermented Rooibos, in particular, was found to improve several dimensions including sperm concentration, viability and motility [38]. These findings imply that Rooibos consumption could have a role to play in supporting male fertility, namely by sequestering oxidative damage by improving antioxidant defence mechanisms and subsequently improving the sperm quality and function [39]. Human randomised controlled trials are now needed to explore this.

Fermented Rooibos extracts have been found to inhibit human basophil activation [22] a finding that supports earlier laboratory research showing that Rooibos has allergen-dependent inhibitory 
Table 1: Rooibos Tea \& Health: Evidence from Human Trials.

\begin{tabular}{|c|c|c|c|c|c|}
\hline $\begin{array}{l}\text { Study (Author- Year- } \\
\text { Location- Reference } \\
\text { Number) }\end{array}$ & $\begin{array}{l}\text { Subjects (age- } \\
\text { gender- number) }\end{array}$ & Study design & $\begin{array}{c}\text { Tea Intervention } \\
\text { (type) }\end{array}$ & Tea Intervention (dosage) & $\begin{array}{l}\text { Main findings (with any } \\
\text { reported significant } \\
\text { p-values) }\end{array}$ \\
\hline $\begin{array}{l}\text { Pedretti s- et al. (2020) } \\
\text { [22] South Africa }\end{array}$ & $\mathrm{n}=9$ atopic adults & $\begin{array}{l}\text { Used ex vivo } \\
\text { samples from } \\
\text { atopic patients }\end{array}$ & $\begin{array}{l}\text { Fermented and } \\
\text { unfermented } \\
\text { Rooibos extracts }\end{array}$ & $\begin{array}{l}\text { Three optimised decreasing } \\
\text { concentrations of unfermented } \\
\text { (0.1- } 0.03 \text { or } 0.01 \mathrm{mg} / \mathrm{ml} \text { ) or } \\
\text { fermented Rooibos extracts } \\
\text { (0.05- } 0.017 \text { or } 0.005 \mathrm{mg} / \\
\mathrm{ml}) \text { were used during the } \\
\text { experiments }\end{array}$ & $\begin{array}{l}\text { Rooibos extracts inhibited } \\
\text { basophil activation in a } \\
\text { dose-dependent non- } \\
\text { allergen specific manner. } \\
\text { The inhibitory effect was } \\
\text { stronger using fermented } \\
\text { versus unfermented extract. }\end{array}$ \\
\hline $\begin{array}{l}\text { Rodgers A- et al. (2016) } \\
\text { [23] South Africa }\end{array}$ & $\begin{array}{l}\mathrm{n}=8 \text { calcium } \\
\text { oxalate }(\mathrm{CaOx}) \\
\text { renal stone } \\
\text { formers }\end{array}$ & 30-day trial & $\begin{array}{l}\text { Green tea from } \\
\text { Japan or Rooibos } \\
\text { from South Africa }\end{array}$ & $\begin{array}{l}\text { Samples were prepared for } \\
\text { analysis by adding } 250 \mathrm{ml} \text { of } \\
\text { boiling water to each teabag. } \\
\text { These were removed after } \\
\text { brewing times of } 5 \text { and } 10 \mathrm{~min} \text {. }\end{array}$ & $\begin{array}{l}\text { Ingestion of Rooibos tea } \\
\text { does not reduce the risk } \\
\text { factors for CaOx stone } \\
\text { formation in humans- }\end{array}$ \\
\hline $\begin{array}{l}\text { Breiter T- et al. (2011) } \\
\text { [20] Germany }\end{array}$ & $\begin{array}{l}\mathrm{n}=12 \text { healthy } \\
\text { males. }\end{array}$ & $\begin{array}{c}\text { 24-hr crossover } \\
\text { trial. }\end{array}$ & $\begin{array}{l}\text { Different Rooibos } \\
\text { drinksfrom } \\
\text { unfermented } \\
\text { Rooibos or a } \\
\text { placebo }\end{array}$ & $\begin{array}{l}\text { 10g Rooibos extracted with } \\
500 \mathrm{ml} \text { boiling water seeped from } \\
10 \text { minutes }\end{array}$ & $\begin{array}{l}\text { On average a total of } \\
0.76 \mathrm{nmol} \text { of flavonoids were } \\
\text { detected during their peak } \\
\text { concentration after intake of } \\
\text { the Rooibos tea- accounting } \\
\text { for } 0.26 \% \text { compared to the } \\
\text { total amount of flavonoids } \\
\text { ingested. }\end{array}$ \\
\hline $\begin{array}{l}\text { Marnewick JL- et al. } \\
\text { (2011) [21] South } \\
\text { Africa }\end{array}$ & $\mathrm{n}=40$ volunteers & 6-week trial & $\begin{array}{c}\text { Fermented/ } \\
\text { traditional Rooibos }\end{array}$ & $\begin{array}{c}\text { Six } 200 \text { ml cups daily (one tea } \\
\text { bag per cup; } 5 \text { minute infusion } \\
\text { time). }\end{array}$ & $\begin{array}{l}\text { Consumption of fermented- } \\
\text { traditional Rooibos } \\
\text { significantly improved the } \\
\text { lipid profile as well as redox } \\
\text { status- both relevant to } \\
\text { heart disease- in adults } \\
\text { at risk for developing } \\
\text { cardiovascular disease. }\end{array}$ \\
\hline $\begin{array}{l}\text { Persson IA- et al. } \\
\text { (2010) [25] Sweden }\end{array}$ & $\begin{array}{l}\mathrm{n}=17 \text { healthy } \\
\text { volunteers. }\end{array}$ & $\begin{array}{l}\text { Three phase } \\
\text { randomized- } \\
\text { three-phase- } \\
\text { crossover study. }\end{array}$ & $\begin{array}{l}\text { Green tea- black } \\
\text { tea or Rooibos tea } \\
\text { (South Africa) }\end{array}$ & $\begin{array}{l}10 \mathrm{~g} \text { tea in } 400 \mathrm{ml} 10 \mathrm{~min} \text { infusion } \\
\text { freshly prepared and cooled so } \\
\text { the participants could drink it } \\
\text { within approximately } 2 \mathrm{~min} \text {. }\end{array}$ & $\begin{array}{l}\text { Oral intake of a single dose } \\
\text { of Rooibos tea significantly } \\
\text { inhibited ACE activity after } \\
30 \mathrm{~min}(\mathrm{p}<0.01) \text { and after } \\
60 \mathrm{~min}(\mathrm{p}<0.05) \text {. Rooibos } \\
\text { tea may have cardiovascular } \\
\text { effects through inhibition of } \\
\text { ACE activity. }\end{array}$ \\
\hline $\begin{array}{l}\text { Utter AC- et al. (2010) } \\
\text { [24] USA }\end{array}$ & $\mathrm{n}=23$ athletes & $\begin{array}{l}\text { Randomized- } \\
\text { cross-over design } \\
\text { with three } \\
\text { different study } \\
\text { arms. } \\
\end{array}$ & $\begin{array}{c}\text { Rooibos tea- } \\
\text { carbohydrate } \\
\text { beverage or bottled } \\
\text { water (placebo) }\end{array}$ & NR (restricted access paper) & $\begin{array}{l}\text { Rooibos tea was no more } \\
\text { effective in promoting } \\
\text { rehydration than plain } \\
\text { water. }\end{array}$ \\
\hline $\begin{array}{l}\text { Stalmach A- et al. } \\
\text { (2009) [14] Italy }\end{array}$ & $n=10$ volunteers & $\begin{array}{l}\text { Bioavailability } \\
\text { trial }\end{array}$ & $\begin{array}{l}\text { Fermented or } \\
\text { unfermented } \\
\text { Rooibos tea }\end{array}$ & $500 \mathrm{ml}$ & $\begin{array}{l}\text { The overall metabolite } \\
\text { levels excreted were } 82 \\
\text { and } 352 \text { nmol- accounting } \\
\text { for } 0.09 \text { and } 0.22 \% \text { of the } \\
\text { flavonoids in the fermented } \\
\text { and unfermented drinks- } \\
\text { respectively. Most of the } \\
\text { aspalathin metabolites } \\
\text { were excreted within } 5 \mathrm{~h} \text { of } \\
\text { tea consumption- implying } \\
\text { absorption in the small } \\
\text { intestine. }\end{array}$ \\
\hline
\end{tabular}

Key: ACE- Angiotensin-Converting Enzyme; NR- Not Reported.

Citation: Bond TJ, Derbyshire EJ (2020) Rooibos Tea and Health: A Systematic Review of the Evidence from the Last Two Decades. 
Table 2: Rooibos Tea and Health: Evidence from Laboratory Studies.

\begin{tabular}{|c|c|c|c|c|}
\hline $\begin{array}{l}\text { Study (Author- } \\
\text { Year- Reference } \\
\text { Number) }\end{array}$ & $\begin{array}{c}\text { Laboratory/ } \\
\text { Mechanistic study }\end{array}$ & $\begin{array}{c}\text { Rooibos tea Intervention } \\
\text { (type) }\end{array}$ & Main outcomes & Main findings \\
\hline $\begin{array}{l}\text { Lawal AO- et al. } \\
\text { (2019) [27] }\end{array}$ & $\begin{array}{l}\text { In vitro antioxidant } \\
\text { capacity }\end{array}$ & $\begin{array}{l}\text { Aqueous extracts of } \\
\text { fermented Rooibos- green } \\
\text { Rooibos and Honey bush. }\end{array}$ & Oxidative stress & $\begin{array}{l}\text { Herbal extracts offered protection against diesel } \\
\text { exhaust particles that induced oxidative stress and } \\
\text { inflammatory response. }\end{array}$ \\
\hline $\begin{array}{l}\text { Mazibuko-Mbejeet } \\
\text { SE- al. (2019) [43] }\end{array}$ & $\begin{array}{l}\text { In vitro- using liver } \\
\text { cells }\end{array}$ & Aspalathin treatment & $\begin{array}{l}\text { Hepatic insulin } \\
\text { resistance }\end{array}$ & $\begin{array}{l}\text { Aspalathin improved insulin signalling and } \\
\text { mitochondrial bioenergetics. }\end{array}$ \\
\hline $\begin{array}{l}\text { Mazibuko-Mbeje } \\
\text { SE- et al. (2019) } \\
{[44]}\end{array}$ & In vitro- using rats & $\begin{array}{l}\text { Aspalathin-enriched green } \\
\text { Rooibos extract }\end{array}$ & $\begin{array}{l}\text { Hepatic insulin } \\
\text { resistance }\end{array}$ & $\begin{array}{c}\text { Green Rooibos extract showed potential in } \\
\text { ameliorating hepatic insulin resistance by improving } \\
\text { insulin sensitivity via PI3K/AKT- FOXO1 and AMPK- } \\
\text { mediated pathways. }\end{array}$ \\
\hline $\begin{array}{l}\text { Morishita Y- et al. } \\
\text { (2019) [26] }\end{array}$ & $\begin{array}{l}\text { Rat basophilic } \\
\text { leukaemia cells }\end{array}$ & $\begin{array}{l}\text { Quercetin- luteolin and } \\
\text { chrysoeriol were mixed } \\
\text { in the ratio that occurs in } \\
\text { Rooibos tea extract }\end{array}$ & Allergic response & $\begin{array}{c}\text { The mixture inhibited antigen- and calcium } \\
\text { ionophore-stimulated degranulation to the same } \\
\text { degree as whole Rooibos tea extract. Flavonoids } \\
\text { underly the degranulation inhibitory activity of } \\
\text { rooibos tea. }\end{array}$ \\
\hline $\begin{array}{l}\text { Orlando P- et al. } \\
\text { (2019) [28] }\end{array}$ & $\begin{array}{l}\text { Diabetic and non- } \\
\text { diabetic vervet } \\
\text { monkeys }\end{array}$ & $\begin{array}{c}90 \mathrm{mg} / \mathrm{kg} \text { of aspalathin-rich } \\
\text { green Rooibos extract for } 28 \\
\text { days. }\end{array}$ & $\begin{array}{l}\text { Oxidative stress- LDL } \\
\text { cholesterol }\end{array}$ & $\begin{array}{c}\text { Green Rooibos extract could counteract hyperglycemia- } \\
\text { oxidative stress and dyslipidemia- lowering } \\
\text { cardiovascular risk factors linked to diabetes. }\end{array}$ \\
\hline $\begin{array}{l}\text { Pyrzanowska J- et } \\
\text { al. (2019) [56] }\end{array}$ & $\begin{array}{l}\text { Sprague-Dawley } \\
\text { male rats }\end{array}$ & $\begin{array}{l}\text { Infusions- prepared using } \\
1-2 \text { and } 4 \mathrm{~g} \text { of 'fermented' } \\
\text { Rooibos leaves for } 100 \mathrm{ml} \text { of } \\
\text { hot water. }\end{array}$ & Spatial memory & $\begin{array}{c}\text { All treated rats showed improvement of long- } \\
\text { term spatial memory. Striatal dopamine and } \\
\text { 3-methoxytyramine levels were increased in treated } \\
\text { rats. }\end{array}$ \\
\hline $\begin{array}{l}\text { Uličná O- et al. } \\
\text { (2019) [12] }\end{array}$ & $\begin{array}{l}\text { Rats with carbon } \\
\text { tetrachloride- } \\
\text { induced liver } \\
\text { damage }\end{array}$ & Rooibos tea administration & $\begin{array}{l}\text { Antioxidant activity- } \\
\quad \text { liver damage }\end{array}$ & $\begin{array}{l}\text { Improved histological features support the view of } \\
\text { antioxidant and membrane-stabilizing activity of } \\
\text { Rooibos tea. This may play a role in the protection of } \\
\text { the liver from injury caused by known toxins. }\end{array}$ \\
\hline $\begin{array}{l}\text { Dludla PV- et al. } \\
\text { (2018) [45] }\end{array}$ & Diabetic mice & Aspalathin intervention & Glycaemic control & $\begin{array}{l}\text { Metformin and a high dose }(130 \mathrm{mg} / \mathrm{kg}) \text { of } \\
\text { aspalathin ameliorated diabetic symptoms i.e. } \\
\text { abnormally raised fasting plasma glucose levels. }\end{array}$ \\
\hline $\begin{array}{l}\text { Moosa S- et al. } \\
(2018)[51]\end{array}$ & Murine study & Rooibos tea extract & Bone health & $\begin{array}{c}\text { Fermented Rooibos had a more potent inhibitory } \\
\text { effect on osteoclasts and associated gene expression } \\
\text { than unfermented Rooibos extract. }\end{array}$ \\
\hline $\begin{array}{l}\text { Yang S- et al. (2018) } \\
\text { [71] }\end{array}$ & Murine study & $\begin{array}{l}\text { Aspalathin and nothofagin } \\
\text { from green Rooibos }\end{array}$ & Sepsis & $\begin{array}{l}\text { Aspalathin and nothofagin appear to protect mice } \\
\text { against sepsis-triggered renal injury. }\end{array}$ \\
\hline $\begin{array}{l}\text { Akinrinmade 0- et } \\
\text { al. (2017) [57] }\end{array}$ & $\begin{array}{l}\text { Adult male Wistar } \\
\text { rats }\end{array}$ & $\begin{array}{c}\text { Fermented Rooibos herbal } \\
\text { tea }\end{array}$ & $\begin{array}{l}\text { Brain oedema- } \\
\text { neuronal apoptosis }\end{array}$ & $\begin{array}{l}\text { Long-term consumption of fermented Rooibos tea } \\
\text { significantly reduced brain oedema and neuronal } \\
\text { apoptosis. }\end{array}$ \\
\hline $\begin{array}{l}\text { Dludla PV- et al. } \\
\text { (2017) [46] }\end{array}$ & Diabetic mice & Aspalathin intervention & Glycaemic control & $\begin{array}{l}\text { Aspalathin maintained cellular homeostasis and } \\
\text { protected the myocardium against hyperglycemia- } \\
\text { induced oxidative stress by activating Nrf2 and its } \\
\text { downstream target genes. }\end{array}$ \\
\hline $\begin{array}{l}\text { Johnson R- et al. } \\
\text { (2017) [72] }\end{array}$ & In vitro & Aspalathin intervention & Cardioprotection & $\begin{array}{l}\text { Aspalathin co-treatment could protect the } \\
\text { myocardium from Dox-induced cardiotoxicity. }\end{array}$ \\
\hline $\begin{array}{l}\text { Johnson R- et al. } \\
\text { (2017) [73] }\end{array}$ & $\begin{array}{l}\text { Cardiomyocyte } \\
\text { model }\end{array}$ & Aspalathin intervention & Cardioprotection & $\begin{array}{l}\text { Aspalathin activated Adipoq and modulated the } \\
\text { expression of Ppary and Srebf1/2- decreasing } \\
\text { inflammation via Il6/Jak2 pathway- which increased } \\
\text { expression of Bcl } 2 \text { preventing myocardium apoptosis. }\end{array}$ \\
\hline
\end{tabular}




\begin{tabular}{|c|c|c|c|c|}
\hline $\begin{array}{l}\text { Ros-Santaella JL- et } \\
\text { al. (2017) [36] }\end{array}$ & Boar semen & $\begin{array}{c}\text { Four concentrations both of } \\
\text { fermented and unfermented } \\
\text { Rooibos extracts }\end{array}$ & Sperm function & $\begin{array}{c}\text { Rooibos extract enhanced sperm velocity- protected } \\
\text { acrosome structure- and preserved membrane } \\
\text { integrity during semen storage. }\end{array}$ \\
\hline $\begin{array}{l}\text { Johnson R- et al. } \\
\text { (2017) [74] }\end{array}$ & In vitro & Aspalathin intervention & Cardioprotection & $\begin{array}{c}\text { Aspalathin increased glucose oxidation and } \\
\text { modulated fatty acid utilization and induced a } \\
\text { favourable substrate shift in } \mathrm{H} 9 \mathrm{c} 2 \text { cardiomyocytes } \\
\text { exposed to high glucose. }\end{array}$ \\
\hline $\begin{array}{l}\text { Nash LA- et al } \\
\text { (2016) [52] }\end{array}$ & Saos 2 cells & $\begin{array}{l}\text { Rooibos- green and black tea } \\
\text {-normalized to } 1 \text { or } 10 \mu \mathrm{g} / \\
\mathrm{mL} \text { gallic acid equivalents. }\end{array}$ & Bone health & $\begin{array}{l}\text { Green- black and Rooibos tea improved osteoblast } \\
\text { activity at a low level. }\end{array}$ \\
\hline $\begin{array}{l}\text { Kamakura R- et al. } \\
\text { (2015) [40] }\end{array}$ & $\begin{array}{l}\text { Obese diabetic } \\
\text { mice }\end{array}$ & Green Rooibos extract & $\begin{array}{l}\text { Blood glucose- } \\
\text { antidiabetic potential }\end{array}$ & $\begin{array}{c}\text { Green Rooibos extract suppressed the increase in } \\
\text { fasting blood glucose levels in type } 2 \text { diabetic model } \\
\text { mice. }\end{array}$ \\
\hline $\begin{array}{l}\text { Ku SK- et al. (2015) } \\
\text { [42] }\end{array}$ & $\begin{array}{l}\text { Human umbilical } \\
\text { vein endothelial } \\
\text { cells and mice }\end{array}$ & $\begin{array}{l}\text { Aspalathin and nothofagin } \\
\text { from green Rooibos }\end{array}$ & $\begin{array}{l}\text { High glucose-induced } \\
\text { inflammation }\end{array}$ & $\begin{array}{c}\text { Treatment of aspalathin or nothofagin inhibited } \\
\text { high glucose-mediated vascular hyperpermeability- } \\
\text { adhesion of monocytes toward human umbilical vein } \\
\text { endothelial cells- and expression of cell adhesion } \\
\text { molecules. }\end{array}$ \\
\hline $\begin{array}{l}\text { Lee W- et al. (2015) } \\
\text { [65] }\end{array}$ & $\begin{array}{l}\text { Human umbilical } \\
\text { vein endothelial } \\
\text { cells and mice }\end{array}$ & $\begin{array}{l}\text { Aspalathin and nothofagin } \\
\text { from green Rooibos }\end{array}$ & $\begin{array}{l}\text { Anti-inflammatory } \\
\text { functions }\end{array}$ & $\begin{array}{c}\text { Aspalathin and nothofagin possess anti-inflammatory } \\
\text { functions are could be a useful therapy for vascular } \\
\text { inflammatory diseases. }\end{array}$ \\
\hline $\begin{array}{l}\text { Mazibuko SE- et al. } \\
\text { (2015) [47] }\end{array}$ & $\begin{array}{l}\text { In vitro- using } \\
\text { cultured } \\
\text { adipocytes }\end{array}$ & $\begin{array}{l}\text { Treated with green Rooibos } \\
\text { extract or aspalathin }\end{array}$ & $\begin{array}{l}\text { Glucose and lipid } \\
\text { metabolism }\end{array}$ & $\begin{array}{l}\text { At a protein level green rooibos extract and } \\
\text { aspalathin suppressed markers of insulin resistance } \\
\text { i.e. insulin receptor substrate one. }\end{array}$ \\
\hline $\begin{array}{l}\text { van der Merwe JD- } \\
\text { et al. (2015) [29] }\end{array}$ & Male Fischer rats & $\begin{array}{l}\text { Aspalathin-enriched green } \\
\text { Rooibos extract }\end{array}$ & Antioxidant activity & $\begin{array}{c}\text { Glutathione reductase activity significantly }(p<0.05) \\
\text { increased after } 28 \text { days- while glutathione content } \\
\text { was decreased after } 90 \text { days- suggesting an altered } \\
\text { glutathione redox cycle. }\end{array}$ \\
\hline $\begin{array}{l}\text { Waisundara \& Hoon } \\
\text { (2015) [30] }\end{array}$ & $\begin{array}{l}\text { In vitro models } \\
\text { of diabetes and } \\
\text { cancer }\end{array}$ & Rooibos tea & $\begin{array}{l}\text { Oxidative stress- } \\
\text { diabetes- cancer }\end{array}$ & $\begin{array}{l}\text { The Rooibos tea extract was observed to have } \\
\text { increased the CAT and SOD activities in two in vitro } \\
\text { disease models. }\end{array}$ \\
\hline $\begin{array}{l}\text { Ajuwon OR- et al. } \\
\text { (2014) [31] }\end{array}$ & Male Wistar rats & Fermented Rooibos extract & $\begin{array}{l}\text { Hepatoprotection- } \\
\text { Oxidative stress }\end{array}$ & $\begin{array}{l}\text { Aqueous Rooibos extract attenuated LPS-induced } \\
\text { liver injury possibly by modulating oxidative stress } \\
\text { and suppressing pro-inflammatory cytokines } \\
\text { formation. }\end{array}$ \\
\hline $\begin{array}{l}\text { Ayeleso AO- et al. } \\
\text { (2014) [37] }\end{array}$ & Diabetic rats & $\begin{array}{l}\text { Aqueous Rooibos tea extract } \\
(2 \%) \text { for } 7 \text { weeks. }\end{array}$ & Sperm function & $\begin{array}{c}\text { Significant }(p<0.05) \text { elevated levels of wobble and } \\
\text { sperm linearity were observed following Rooibos tea } \\
\text { extract treatment }\end{array}$ \\
\hline $\begin{array}{l}\text { Canda BD- et al. } \\
\text { (2014) [32] }\end{array}$ & $\begin{array}{l}\text { Forty male Wistar } \\
\text { rats }\end{array}$ & $\begin{array}{l}\text { Fermented Rooibos- } \\
\text { unfermented rooibos- a } \\
\text { rooibos-derived commercial } \\
\text { supplement- or water. }\end{array}$ & $\begin{array}{l}\text { Oxidative stress- } \\
\text { Antioxidant activity }\end{array}$ & $\begin{array}{l}\text { Fermented Rooibos caused a decrease }(p<0.05) \text { in } \\
\text { superoxide dismutase activity. }\end{array}$ \\
\hline $\begin{array}{l}\text { Dludla PV- et al. } \\
\text { (2014) [33] }\end{array}$ & Diabetic rats & $\begin{array}{l}\text { Aqueous extract of } \\
\text { fermented Rooibos }\end{array}$ & Oxidative stress & $\begin{array}{l}\text { Aqueous extract of fermented Rooibos protected } \\
\text { cardiomyocytes- derived from diabetic rats- against } \\
\text { experimentally induced oxidative stress and } \\
\text { ischemia. }\end{array}$ \\
\hline $\begin{array}{l}\text { Hong IS- et al. } \\
\text { (2014) [34] }\end{array}$ & Laboratory model & Rooibos tea & Oxidative stress & $\begin{array}{l}\text { Rooibos tea appears to (i) reverse the increase } \\
\text { in stress-related metabolites (ii) prevent lipid } \\
\text { peroxidation- (iii) restore stress-induced protein } \\
\text { degradation- (iv) regulate glutathione metabolism } \\
\text { and (v) modulate changes in the activities of } \\
\text { antioxidant enzymes. }\end{array}$ \\
\hline
\end{tabular}




\begin{tabular}{|c|c|c|c|c|}
\hline $\begin{array}{l}\text { Opuwari CS- et al. } \\
\text { (2014) [38] }\end{array}$ & Male rats & Fermented Rooibos & Sperm function & $\begin{array}{c}\text { Fermented Rooibos significantly improved sperm } \\
\text { vitality }(p<0.01) \text { - but caused a significant increase in } \\
\text { spontaneous acrosome reaction }(p<0.05) \text { - whereas } \\
\text { unfermented did not. }\end{array}$ \\
\hline $\begin{array}{l}\text { Sanderson M- et al. } \\
(2014)[50]\end{array}$ & In vitro & Fermented Rooibos & Obesity prevention & $\begin{array}{l}\text { Hot water soluble solids from fermented Rooibos } \\
\text { inhibited adipogenesis- suggesting its potential in } \\
\text { preventing obesity. }\end{array}$ \\
\hline $\begin{array}{l}\text { Schloms L- et al. } \\
\text { (2014) [75] }\end{array}$ & Rats & Rooibos & Inflammation & $\begin{array}{l}\text { In vivo studies demonstrate that Rooibos significantly } \\
\text { decreased glucocorticoid levels in rats and steroid } \\
\text { metabolite ratios linked to metabolic disorders. }\end{array}$ \\
\hline $\begin{array}{l}\text { Mazibuko SE- et al. } \\
\text { (2013) [76] }\end{array}$ & $\begin{array}{l}\text { In vitro using } \\
\text { skeletal muscle } \\
\text { cells }\end{array}$ & $\begin{array}{l}\text { Treated with aspalathin- } \\
\text { enriched green } \\
\text { (unfermented) Rooibos } \\
\text { extract }\end{array}$ & Insulin resistance & $\begin{array}{l}\text { Rooibos attenuated palmitate-induced insulin } \\
\text { resistance in } C_{2} C_{12} \text { skeletal muscle cells. }\end{array}$ \\
\hline $\begin{array}{l}\text { Awoniyi DO- et al. } \\
\text { (2012) [39] }\end{array}$ & Male Wistar rats & $\begin{array}{l}\text { Fermented Rooibos- 'green' } \\
\text { Rooibos- Chinese green tea- } \\
\text { Rooibos supplement- green } \\
\text { tea supplement or water. }\end{array}$ & Sperm function & $\begin{array}{l}\text { Both Rooibos extracts offered a measure of } \\
\text { protection against induced oxidative damage by } \\
\text { increasing the antioxidant defence mechanisms } \\
\text { improving the sperm quality and function. }\end{array}$ \\
\hline $\begin{array}{l}\text { Muller CJ- et al. } \\
\text { (2012) [41] }\end{array}$ & In vitro & $\begin{array}{l}\text { Rooibos extract high in } \\
\text { aspalathin content }\end{array}$ & $\begin{array}{l}\text { Hypoglycaemic } \\
\text { potential }\end{array}$ & $\begin{array}{l}\text { In vivo the extract sustained a glucose lowering } \\
\text { effect comparable to metformin over a } 6 \mathrm{~h} \text { period } \\
\text { after administration ( } 25 \mathrm{mg} / \mathrm{kg} \text { body weight }(\mathrm{BW}) \text { ) to } \\
\text { STZ-induced diabetic rats. }\end{array}$ \\
\hline $\begin{array}{l}\text { Pantsi WG- et al. } \\
\text { (2011) [77] }\end{array}$ & Male Wistar rats & $\begin{array}{c}\text { Aqueous Rooibos and green } \\
\text { tea }\end{array}$ & Cardioprotection & $\begin{array}{c}\text { Results demonstrate the cardio-protective properties } \\
\text { of aqueous Rooibos extracts via the inhibition } \\
\text { of apoptosis which can possibly be related to its } \\
\text { flavonol content. }\end{array}$ \\
\hline $\begin{array}{l}\text { Sissing O- et al. } \\
(2011)[58]\end{array}$ & Male rats & $\begin{array}{l}\text { Rooibos- Honeybush and } \\
\text { Camellia sinensis teas }\end{array}$ & $\begin{array}{l}\text { Esophageal } \\
\text { papillomas }\end{array}$ & $\begin{array}{l}\text { The mean total papilloma size was reduced by } \\
\text { unfermented Rooibos ( } 87 \%) \text {. }\end{array}$ \\
\hline $\begin{array}{l}\text { Hendricks R- et al. } \\
\text { (2010) [54] }\end{array}$ & $\begin{array}{l}\text { Whole blood } \\
\text { culture assays }\end{array}$ & $\begin{array}{l}\text { Rooibos tea and Camellia } \\
\text { sinensis }\end{array}$ & Immune function & $\begin{array}{l}\text { Rooibos and black tea modulate immune function in } \\
\text { vitro. Rooibos tea stimulated whole blood cultures } \\
\text { induced higher Interleukin-6- lower Interleukin-10- } \\
\text { and had no effect on Interferon gamma secretion }\end{array}$ \\
\hline $\begin{array}{l}\text { Baba H- et al. } \\
(2009)[10]\end{array}$ & $\begin{array}{l}\text { Seven-week-old } \\
\text { Wister rats }\end{array}$ & Rooibos tea and water. & $\begin{array}{l}\text { Oxidative stress- } \\
\text { Antioxidant activity }\end{array}$ & $\begin{array}{l}\text { Serum SOD levels were significantly higher in the } \\
\text { Rooibos group compared to the controls }(p<0.05) \text {. }\end{array}$ \\
\hline $\begin{array}{l}\text { Kawano A- et al. } \\
\text { (2009) [48] }\end{array}$ & $\begin{array}{l}\text { Type } 2 \text { diabetes } \\
\text { model mice in vivo }\end{array}$ & $\begin{array}{l}\text { Aspalathin- a green Rooibos } \\
\text { tea component }\end{array}$ & $\begin{array}{l}\text { Antidiabetic } \\
\text { potential }\end{array}$ & $\begin{array}{l}\text { Aspalathin appears to have beneficial effects } \\
\text { on glucose homeostasis in type } 2 \text { diabetes by } \\
\text { stimulating glucose uptake in muscle tissues and } \\
\text { insulin secretion from pancreatic beta-cells. }\end{array}$ \\
\hline $\begin{array}{l}\text { Marnewick JL- et al. } \\
\text { (2009) [61] }\end{array}$ & Rat liver & $\begin{array}{l}\text { Unfermented and fermented } \\
\text { Rooibos }\end{array}$ & Chemo protection & $\begin{array}{l}\text { Unfermented rooibos significantly }(p<0.05) \text { to } \\
\text { marginally }(p<0.1) \text { reduced the total number of foci } \\
(>10 \text { microm). Fermentation seems to reduce the } \\
\text { protective effect of the herbal teas. }\end{array}$ \\
\hline $\begin{array}{l}\text { Gilani AH- et al. } \\
\text { (2006) [59] }\end{array}$ & $\begin{array}{l}\text { Isolated tissue } \\
\text { preparations }\end{array}$ & $\begin{array}{c}\text { Aqueous extract of Rooibos } \\
\text { tea }\end{array}$ & $\begin{array}{l}\text { Antispasmodic } \\
\text { effects }\end{array}$ & $\begin{array}{c}\text { Rooibos tea possessed a combination of dominant } \mathrm{K} \\
\text { (ATP) channel activation and weak } \mathrm{Ca}(++) \text { antagonist } \\
\text { mechanisms justifying its use in hyperactive } \\
\text { gastrointestinal disorders. }\end{array}$ \\
\hline $\begin{array}{l}\text { Khan AU- et al. } \\
(2006)[60]\end{array}$ & $\begin{array}{l}\text { Isolated tissue } \\
\text { preparations }\end{array}$ & $\begin{array}{c}\text { Aqueous extract of Rooibos } \\
\text { tea }\end{array}$ & $\begin{array}{l}\text { Bronchodilator- } \\
\text { antispasmodic }\end{array}$ & $\begin{array}{l}\text { The bronchodilator- antispasmodic and blood } \\
\text { pressure lowering effects of Rooibos tea appear to } \\
\text { be mediated predominantly through K (ATP) channel } \\
\text { activation with the selective bronchodilatory effect. }\end{array}$ \\
\hline
\end{tabular}




\begin{tabular}{|c|c|c|c|c|}
\hline $\begin{array}{l}\text { Ulicná O- et al. } \\
\text { (2006) [49] }\end{array}$ & $\begin{array}{l}\text { Streptozotocin- } \\
\text { induced diabetic } \\
\text { rats }\end{array}$ & $\begin{array}{l}\text { Aqueous and alkaline } \\
\text { extracts of Rooibos tea }\end{array}$ & $\begin{array}{l}\text { Antidiabetic } \\
\text { potential }\end{array}$ & $\begin{array}{l}\text { Antioxidant compounds in rooibos tea partially } \\
\text { prevent oxidative stress and they are effective in } \\
\text { both hydrophobic and hydrophilic biological systems. }\end{array}$ \\
\hline $\begin{array}{l}\text { van der Merwe JD- } \\
\text { et al. (2006) [78] }\end{array}$ & $\begin{array}{c}\text { Comparative- in } \\
\text { vitro }\end{array}$ & $\begin{array}{l}\text { Rooibos and honey bush tea } \\
\text { compared with black- oolong } \\
\text { and green teas }\end{array}$ & Antimutagenic & $\begin{array}{l}\text { Antimutagenic activity of the South African herbal } \\
\text { teas was mutagen-specific- affected by fermentation. } \\
\text { Unfermented Rooibos was less effective than } \\
\text { fermented Rooibos }\end{array}$ \\
\hline $\begin{array}{l}\text { Lee EJ- et al. (2004) } \\
\text { [79] }\end{array}$ & $\begin{array}{l}\text { DNA strand } \\
\text { scission }\end{array}$ & Rooibos tea & Antioxidant activity & $\begin{array}{l}\text { Result suggests that total soluble phenolics- specially } \\
\text { flavonoid- of Rooibos tea are responsible for several } \\
\text { kinds of antioxidant activities and preventive activity } \\
\text { on peroxyl radical induced DNA strand scission. }\end{array}$ \\
\hline $\begin{array}{l}\text { Kucharská J- et al. } \\
\text { (2004) [35] }\end{array}$ & $\begin{array}{l}\text { Rat model } \\
\text { of carbon } \\
\text { tetrachloride- } \\
\text { induced liver } \\
\text { damage }\end{array}$ & Rooibos tea & Oxidative stress & $\begin{array}{l}\text { Rooibos tea restored liver concentrations of CoQ9H2 } \\
\text { and alpha-tocopherol and inhibited the formation of } \\
\text { MDA. }\end{array}$ \\
\hline $\begin{array}{l}\text { Marnewick JL- et al. } \\
\text { (2004) [62] }\end{array}$ & Male Fischer rats & $\begin{array}{l}\text { Unprocessed (not oxidized)- } \\
\text { processed (oxidized) rooibos- } \\
\text { honey bush- green and black } \\
\text { teas }\end{array}$ & Mutagenic response & $\begin{array}{l}\text { The mutagenic response of aflatoxin B1 against } \\
\text { Salmonella strain TA } 100 \text { was significantly }(p<0.05) \\
\text { inhibited by cytosolic fractions from rats treated with } \\
\text { processed and unprocessed herbal teas. }\end{array}$ \\
\hline $\begin{array}{l}\text { Ulicná O- et al. } \\
\text { (2003) [53] }\end{array}$ & $\begin{array}{c}\text { Rat model of liver } \\
\text { injury }\end{array}$ & Rooibos tea & Hepatoprotection & $\begin{array}{c}\text { Rooibos tea showed histological regression of } \\
\text { steatosis and cirrhosis in the liver tissue with a } \\
\text { significant inhibition of the increase of liver tissue } \\
\text { concentrations of malondialdehyde- triacylglycerols } \\
\text { and cholesterol. }\end{array}$ \\
\hline $\begin{array}{l}\text { Kunishiro K- at al. } \\
\text { (2001) [55] }\end{array}$ & In vitro and in vivo. & Rooibos tea extract & Immune function & $\begin{array}{l}\text { Rooibos tea extract may facilitate the antigen- } \\
\text { specific antibody production through selective } \\
\text { augmentation of IL-2 generation both in vitro and in } \\
\text { vivo. }\end{array}$ \\
\hline
\end{tabular}

Key: ATP-Adenosine Triphosphate; CAT-Chloramphenicol Acetyltransferase; DNA-Deoxyribonucleic Acid; IL-Interleukin; LDL-Low-Density Lipoprotein; LPS-Lipopolysaccharide; MDA- Malondialdehyde; ROS- Reactive Oxygen Species- SOD-Superoxide Dismutase; STZ- Streptozotocin.

effects [26]. This research suggests promise for conditions such as allergic rhinitis [22] but further investigations are needed. Additionally, its effects on bone health [51,52], cognitive health $[56,57]$ and potential hepatoprotective effects [12,31,53] warrant further exploration in the form of human trials. Recently, a review of experimental evidence [68] concluded that blood glucose levels were significantly lower in diabetic rodent models treated with rooibos extracts providing phenolic compounds. Other work suggests that Rooibos extracts and its constituent C-glucosyl flavonoids, and Z-2( $\beta$-D-glucopyranosyloxy)-3-phenylpropenoic acid may elicit positive effects on cellular oxidative stress, inflammation and transcription factors that control genes regulating glucose and lipid metabolism helping to attenuate features of metabolic disease [69].

In the present review human subjects tended to drink Rooibos beverages after they had been infused for approximately 10 minutes $[20,23,25]$. Recently, from a consumer stance the ideal 'optimal cup' of Rooibos tea has been defined as one that had been seeped for 10 minutes or longer [70]. Unfortunately, recent research shows that only $15.9 \%$ of respondents consumed an 'optimal cup' of Rooibos in an amount that enables its potential cardioprotective health benefits to be provided (defined as a minimum of 4 to 6 cups per day) [70]. Interestingly, compared with cold and regular-brewed Rooibos infusions of unfermented/green and red Rooibos prepared by boiling have the highest antioxidant capacities and total polyphenol profiles
[9]. Such findings suggest that more information needs to be imparted to consumers' regarding preparation methods and the potential health benefits that drinking Rooibos tea could bring.

With regard to limitations and future research directions greater uniformity is needed in forthcoming studies. For example, some studies used fermented and others unfermented Rooibos extracts or infusions the fermented form appeared to have stronger impact on some health effects $[38,51]$. Others used Rooibos tea and Rooibos 'extracts' or unfermented/green Rooibos. Production seasons and grade quality could also alter the phenolic profiles and antioxidant capacity of Rooibos samples and should be considered [66]. Future trials should also better encompass how findings could be translated into public health messages. For example, Marnewick JL, et al. (2011) [21] asked volunteers to drinks six cups Rooibos tea daily for 6-weeks to study its cardiovascular effects. Similar studies are now needed for other health outcomes such as bone health, liver or brain (cognitive) function.

Considering such evidence Rooibos tea, like teas from Camellia sinensis (e.g. black, green and oolong tea) could be regarded as a 'general health' tea, potentially providing an array of benefits to human health and wellbeing [56] Rooibos tea is naturally slightly sweet [16] thus does not require the addition of extra sugar or artificial sweeteners. It is also caffeine free with a favourable phenolic composition $[9,10]$. (Table 2) 


\section{Conclusions}

In conclusion previous health research has tended to focus on black or green tea. Rooibos is widely consumed in South Africa but is gaining popularity globally. This has been attributed to the fact that it is caffeine free, naturally sweet and abundant in polyphenols with potent antioxidant properties. Now, a growing body of evidence from 7 human studies and 49 laboratory studies suggests that Rooibos could be regarded as a 'general' health tea. Evidence for cardioprotective effects (especially lipid profile) looks promising, particularly as a potential adjunctive therapy. It is suggested that future research now builds on the other potential aspects of health including glycaemic, bone, liver and cognitive wellness enhancing effects that also appear to be emerging.

\section{Disclosure}

The views expressed are those of the authors alone and personnel from the UK TEA \& INFUSIONS ASSOCIATION (UKTIA) had no role in writing this review.

\section{Conflicts of Interest}

The authors declare no conflicts of interest.

\section{Acknowledgements}

The authors received funding provided by the Tea Advisory Panel which is supported by an unrestricted educational grant from the UK TEA \& INFUSIONS ASSOCIATION (UKTIA), the trade association for the UK tea industry. UKTIA plays no role in producing the outputs of the panel. Independent panel members include nutritionists, biochemists, dietitians, dentist and doctors. See www. teaadvisorypanel.com

\section{References}

1. Whitty CJM, MacEwen C, Goddard A, Alderson D, Marshall M, et al. (2020) Rising to the challenge of multimorbidity. BMJ 368: 16964.

2. Doughty KN, Del Pilar NX, Audette A, Katz DL (2017) Lifestyle Medicine and the Management of Cardiovascular Disease. Curr Cardiol Rep 19: 116.

3. Yi M, Wu X, Zhuang W, Xia L, Chen Y, et al. (2019) Tea Consumption and Health Outcomes: Umbrella Review of Meta-Analyses of Observational Studies in Humans. Mol Nutr Food Res 63: e1900389.

4. Hayat K, Iqbal H, Malik U, Bilal U, Mushtaq S (2015) Tea and its consumption: Benefits and Risks. Crit Rev Food Sci Nutr 55: 939-954.

5. Fukasawa R, Kanda A, Hara S (2009) Anti-oxidative Effects of Rooibos Tea Extract on Autoxidation and Thermal Oxidation of Lipids. J Oleo Sci 58: 275-283.

6. Beelders T, Kalili KM, Joubert E, de Beer D, de Villiers A (2012) Comprehensive Two-Dimensional Liquid Chromatographic Analysis of Rooibos (Aspalathus linearis) Phenolics. J Sep Sci 35: 1808-1820.

7. Johnson R, de Beer D, Dludla PV, Ferreira D, Muller CJF, et al. (2018) Aspalathin from Rooibos (Aspalathus linearis): Aspalathin From Rooibos (Aspalathus linearis): A Bioactive C-glucosyl Dihydrochalcone with Potential to Target the Metabolic Syndrome. Planta Med 84: 568-583.

8. Rooibos Council (2016) Latest tea-consumption figues tip scale in favour of rooibos.

9. Damiani E, Carloni P, Rocchetti G, Senizza B, Tianoet L, et al. (2019) Impact of Cold versus Hot Brewing on the Phenolic Profile and Antioxidant Capacity of Rooibos (Aspalathus linearis) Herbal Tea. Antioxidants (Basel) 8: 499.
10. Baba H, Ohtsuka Y, Haruna H, Lee T, Nagata S, et al. (2009) Studies of Anti-Inflammatory Effects of Rooibos Tea in rats. Pediatr Int 51: 700-704.

11. McKay DL, Blumberg JB (2007) A Review of the Bioactivity of South African Herbal Teas: Rooibos (Aspalathus linearis) and Honeybush (Cyclopia intermedia). Phytother Res 21: 1-16.

12. Ulicna O, Vancova O, Kucharska J, Janega P, Waczulíková I (2019) Rooibos Tea (Aspalathus linearis) Ameliorates the $\mathrm{CCl} 4$-induced Injury to Mitochondrial Respiratory Function and Energy Production in Rat Liver. Gen Physiol Biophys 38: 15-25.

13. Stander MA, Van Wyk BE, Taylor MJC, Long HS (2017) Analysis of Phenolic Compounds in Rooibos Tea (Aspalathus linearis) With a Comparison of Flavonoid-Based Compounds in Natural Populations of Plants From Different Regions. J Agric Food Chem 65: 1027010281.

14. Stalmach A, Mullen W, Pecorari M, Serafini M, Crozier A (2009) Bioavailability of C-linked Dihydrochalcone and Flavanone Glucosides in Humans Following Ingestion of Unfermented and Fermented Rooibos Teas. J Agric Food Chem 57: 7104-7111.

15. Erickson L (2003) Rooibos Tea: Research into Antioxidant and Antimutagenic Properties. J Am Botan Council 59: 34-45.

16. Koch IS, Muller M, Joubert E, van der Rijst M, Næsc T (2012) Sensory characterization of rooibos tea and the development of a rooibos sensory wheel and lexicon. Food Res Int 46: 217-28.

17. Joubert E, Gelderblom WC, Louw A, de Beer D (2008) South African Herbal Teas: Aspalathus linearis, Cyclopia spp. and Athrixia Phylicoides--A Review. J Ethnopharmacol 119: 376-412.

18. Chen W, Sudji IR, Wang E, Joubert E, van Wyk BE, et al. (2013) Ameliorative Effect of Aspalathin From Rooibos (Aspalathus linearis) on Acute Oxidative Stress in Caenorhabditis elegans. Phytomedicine 20: 380-386.

19. Gaggia F, Baffoni L, Galiano M, Nielsen DS, Jakobsen RR, et al. (2018) Kombucha Beverage from Green, Black and Rooibos Teas: A Comparative Study Looking at Microbiology, Chemistry and Antioxidant Activity. Nutrients 11: 1.

20. Breiter T, Laue C, Kressel G, Gröll S, Engelhardt UH, et al. (2011) Bioavailability and Antioxidant Potential of Rooibos Flavonoids in Humans Following the Consumption of Different Rooibos Formulations. Food Chem 128: 338-347.

21. Marnewick JL, Rautenbach F, Venter I, Neethling H, Blackhurst DM, et al. (2011) Effects of Rooibos (Aspalathus linearis) on Oxidative Stress and Biochemical Parameters in Adults at Risk for Cardiovascular Disease. J Ethnopharmacol 133: 46-52.

22. Pedretti S, Peter J (2020) Rooibos Herbal Tea Reduces Allergic Basophil Activation in Adult Atopic Patients. J Ethnopharmacol 252: 112353.

23. Rodgers A, Mokoena M, Durbach I, Lazarus J, de Jager $S$, et al. (2016) Do Teas Rich in Antioxidants Reduce the Physicochemical and Peroxidative Risk Factors for Calcium Oxalate Nephrolithiasis in Humans? Pilot Studies With Rooibos Herbal Tea and Japanese Green Tea. Urolithiasis 44: 299-310.

24. Utter AC, Quindry JC, Emerenziani GP, Valiente JS (2010) Effects of Rooibos Tea, Bottled Water, and a Carbohydrate Beverage on Blood and Urinary Measures of Hydration After Acute Dehydration. Res Sports Med 18: 85-96. 
25. Persson IA, Persson K, Hagg S, Andersson RGG (2010) Effects of Green Tea, Black Tea and Rooibos Tea on Angiotensin-Converting Enzyme and Nitric Oxide in Healthy Volunteers. Public Health Nutr 13: $730-737$.

26. Morishita Y, Ikeda K, Matsuno H, Ito H, Tai A (2019) Identification of Degranulation Inhibitors From Rooibos (Aspalathus linearis) Tea in Rat Basophilic Leukaemia Cells. Nat Prod Res 33: 1472-1476.

27. Lawal AO, Davids LM, Marnewick JL (2019) Rooibos (Aspalathus linearis) and Honeybush (Cyclopia Species) Modulate the Oxidative Stress Associated Injury of Diesel Exhaust Particles in Human Umbilical Vein Endothelial Cells. Phytomedicine 59: 152898.

28. Orlando P, Chellan N, Louw J, Tiano L, Cirilli I, et al. (2019) AspalathinRich Green Rooibos Extract Lowers LDL-Cholesterol and Oxidative Status in High-Fat Diet-Induced Diabetic Vervet Monkeys. Molecules 24: 1713.

29. van der Merwe JD, de Beer D, Joubert E, Gelderblom WCA (2015) Short-Term and Sub-Chronic Dietary Exposure to AspalathinEnriched Green Rooibos (Aspalathus linearis) Extract Affects Rat Liver Function and Antioxidant Status. Molecules 20: 22674-22690.

30. Waisundara VY, Hoon LY (2015) Free Radical Scavenging Ability of Aspalathus Linearis in Two in Vitro Models of Diabetes and Cancer. J Tradit Complement Med 5: 174-178.

31. Ajuwon OR, Oguntibeju OO, Marnewick JL (2014) Amelioration of Lipopolysaccharide-Induced Liver Injury by Aqueous Rooibos (Aspalathus linearis) Extract via Inhibition of Pro-Inflammatory Cytokines and Oxidative Stress. BMC Complement Altern Med 14: 392.

32. Canda BD, Oguntibeju OO, Marnewick JL (2014) Effects of Consumption of Rooibos (Aspalathus linearis) and a RooibosDerived Commercial Supplement on Hepatic Tissue Injury by TertButyl Hydroperoxide in Wistar Rats. Oxid Med Cell Longev 2014 716832.

33. Dludla PV, Muller CJ, Louw J, Joubert E, Salie R, et al. (2014) The Cardioprotective Effect of an Aqueous Extract of Fermented Rooibos (Aspalathus linearis) on Cultured Cardiomyocytes Derived From Diabetic Rats. Phytomedicine 21: 595-601.

34. Hong IS, Lee HY, Kim HP (2014) Anti-oxidative Effects of Rooibos Tea (Aspalathus linearis) on Immobilization-Induced Oxidative Stress in Rat Brain. PLoS One 9: e87061.

35. Kucharská J, Ulicná O, Gvozdjáková A, Sumbalová Z, Vancová O, et al. (2004) Regeneration of Coenzyme Q9 Redox State and Inhibition of Oxidative Stress by Rooibos Tea (Aspalathus linearis) Administration in Carbon Tetrachloride Liver Damage. Physiol Res 53: 515-521.

36. Ros-Santaella JL, Pintus E (2017) Rooibos (Aspalathus linearis) Extract Enhances Boar Sperm Velocity Up to 96 Hours of Semen Storage. PLoS One 12: e0183682.

37. Ayeleso AO, Oguntibeju OO, Aboua YG, Brooks NL (2014) Effects of Red Palm Oil and Rooibos on Sperm Motility Parameters in Streptozotocin-Induced Diabetic Rats. Afr J Tradit Complement Altern Med 11: 8-15.

38. Opuwari CS, Monsees TK (2014) In Vivo Effects of Aspalathus linearis (Rooibos) on Male Rat Reproductive Functions. Andrologia 46: 867 877.

39. Awoniyi DO, Aboua YG, Marnewick J, Brooks N (2012) The Effects of Rooibos (Aspalathus linearis), Green Tea (Camellia sinensis) and Commercial Rooibos and Green Tea Supplements on Epididymal Sperm in Oxidative Stress-Induced Rats. Phytother Res 26: 12311239.
40. Kamakura R, Son MJ, de Beer D, Joubert E, Miura Y, et al. (2015) Antidiabetic Effect of Green Rooibos (Aspalathus linearis) Extract in Cultured Cells and Type 2 Diabetic Model KK-A(y) Mice. Cytotechnology 67: 699-710.

41. Muller CJ, Joubert E, de Beer D, Sanderson M, Malherbe CJ, et al. (2012) Acute Assessment of an Aspalathin-Enriched Green Rooibos (Aspalathus linearis) Extract With Hypoglycemic Potential. Phytomedicine 20: 32-39.

42. Ku SK, Kwak S, Kim Y, Bae JS (2015) Aspalathin and Nothofagin From Rooibos (Aspalathus linearis) Inhibits High Glucose-Induced Inflammation in Vitro and in Vivo. Inflammation 38: 445-455.

43. Mazibuko-Mbeje SE, Dludla PV, Johnson R, Joubert E, Louw J, et al. (2019) Aspalathin, a Natural Product With the Potential to Reverse Hepatic Insulin Resistance by Improving Energy Metabolism and Mitochondrial Respiration. PLoS One 14: e0216172.

44. Mazibuko-Mbeje SE, Dludla PV, Roux C, Ghoor S, Joubert E, et al. (2019) Aspalathin-Enriched Green Rooibos Extract Reduces Hepatic Insulin Resistance by Modulating PI3K/AKT and AMPK Pathways. Int J Mol Sci 20: 633.

45. Dludla PV, Gabuza KB, Muller CJF, Joubert E, Louw J, et al. (2018) Aspalathin, a C-glucosyl Dihydrochalcone From Rooibos Improves the Hypoglycemic Potential of Metformin in Type 2 Diabetic (Db/ Db) Mice. Physiol Res 67: 813-818.

46. Dludla PV, Muller CJ, Joubert E, Louw J, Essop MF, et al. (2017) Aspalathin Protects the Heart Against Hyperglycemia-Induced Oxidative Damage by Up-Regulating Nrf2 Expression. Molecules 22: 129.

47. Mazibuko SE, Joubert E, Johnson R, Louw J, Opoku AR, et al. (2015) Aspalathin Improves Glucose and Lipid Metabolism in 3T3-L1 Adipocytes Exposed to Palmitate. Mol Nutr Food Res 59: 2199-2208.

48. Kawano A, Nakamura H, Hata SI, Minakawa M, Miura Y, et al. (2009) Hypoglycemic Effect of Aspalathin, a Rooibos Tea Component From Aspalathus linearis, in Type 2 Diabetic Model Db/Db Mice. Phytomedicine 16: 437-443.

49. Ulicná O, Vancová O, Bozek P, Cársky J, Sebeková K, et al. (2006) Rooibos Tea (Aspalathus linearis) Partially Prevents Oxidative Stress in Streptozotocin-Induced Diabetic Rats Physiol Res 55:157-164.

50. Sanderson M, Mazibuko SE, Joubert E, de Beer D, Johnson R, et al. (2014) Effects of Fermented Rooibos (Aspalathus linearis) on Adipocyte Differentiation. Phytomedicine 21: 109-117.

51. Moosa S, Kasonga AE, Deepak V, Marais S, Magoshi BI, et al. (2018) Rooibos Tea Extracts Inhibit Osteoclast Formation and Activity Through the Attenuation of NF-KB Activity in RAW264.7 Murine Macrophages. Food Funct 9: 3301-3312.

52. Nash LA, Ward WE (2016) Comparison of Black, Green and Rooibos Tea on Osteoblast Activity. Food Funct 7: 1166-1175.

53. Ulicna O, Greksak M, Vancova O, L Zlatos, S Galbavý, et al. (2003) Hepatoprotective Effect of Rooibos Tea (Aspalathus linearis) on CCl4-induced Liver Damage in Rats. Physiol Res 52: 461-466.

54. Hendricks R, Pool EJ (2013) The in Vitro Effects of Rooibos and Black Tea on Immune Pathways. J Immunoassay Immunochem 31: 169180.

55. Kunishiro K, Tai A, Yamamoto I (2001) Effects of Rooibos Tea Extract on Antigen-Specific Antibody Production and Cytokine Generation in Vitro and in Vivo. Biosci Biotechnol Biochem 65: 2137-2145. 
56. Pyrzanowska J, Fecka I, Mirowska-Guzel D, Joniec-Maciejak I, Blecharz-Klin K, et al. (2019) Long-term Administration of Aspalathus linearis Infusion Affects Spatial Memory of Adult Sprague-Dawley Male Rats as Well as Increases Their Striatal Dopamine Content. J Ethnopharmacol 238: 111881.

57. Akinrinmade O, Omoruyi S, Dietrich D, Ekpo O (2017) Longterm Consumption of Fermented Rooibos Herbal Tea Offers Neuroprotection Against Ischemic Brain Injury in Rats. Acta Neurobiol Exp (Wars) 77: 94-105.

58. Sissing L, Marnewick J, de Kock M, Swanevelder S, Joubert E, et al. (2011) Modulating Effects of Rooibos and Honeybush Herbal Teas on the Development of Esophageal Papillomas in Rats. Nutr Cancer 63: $600-610$

59. Gilani AH, Khan AU, Ghayur MN, Ali SF, Herzig (2006) Antispasmodic Effects of Rooibos Tea (Aspalathus linearis) is Mediated Predominantly through $\mathrm{K}^{+}$-Channel Activation. Basic Clin Pharmacol Toxicol 99: 365-373.

60. Khan AU, Gilani AH (2006) Selective Bronchodilatory Effect of Rooibos Tea (Aspalathus linearis) and Its Flavonoid, Chrysoeriol. Eur J Nutr 45: 463-469.

61. Marnewick JL, van der Westhuizen FH, Joubert E, Swanevelder S, Swart P, et al. (2009) Chemoprotective Properties of Rooibos (Aspalathus linearis), Honeybush (Cyclopia intermedia) Herbal and Green and Black (Camellia sinensis) Teas Against Cancer Promotion Induced by Fumonisin B1 in Rat Liver. Food Chem Toxicol 47: 220 229.

62. Marnewick JL, Batenburg W, Swart P, Joubert E, Swanevelder S, et al. (2004) Ex vivo Modulation of Chemical-Induced Mutagenesis by Subcellular Liver Fractions of Rats Treated With Rooibos (Aspalathus linearis) Tea, Honeybush (Cyclopia intermedia) Tea, as Well as Green and Black (Camellia sinensis) Teas. Mutat Res 558: 145-154.

63. Smith C, Swart A (2018) Aspalathus linearis (Rooibos)-A Functional Food Targeting Cardiovascular Disease. Food Funct 9: 5041-5058.

64. Dludla PV, Joubert E, Muller CJF, Louw J, Johnson R (2017) Hyperglycemia-induced Oxidative Stress and Heart DiseaseCardioprotective Effects of Rooibos Flavonoids and Phenylpyruvic acid-2- O-ß-D-glucoside. Nutr Metab (Lond) 14: 45.

65. Lee W, Bae JS (2015) Anti-inflammatory Effects of Aspalathin and Nothofagin from Rooibos (Aspalathus linearis) In Vitro and In Vivo. Inflammation 38: 1502-1516.

66. Joubert E, Beelders T, de Beer D, Malherbe CJ, de Villiers AJ, et al. (2012) Variation in Phenolic Content and Antioxidant Activity of Fermented Rooibos Herbal Tea Infusions: Role of Production Season and Quality Grade. J Agric Food Chem 60: 9171-9179.
67. Darvesh AS, Carroll RT, Bishayee A Geldenhuys WJ, Van der Schyf CJ (2010) Oxidative Stress and Alzheimer's Disease: Dietary Polyphenols as Potential Therapeutic Agents. Expert Rev Neurother 10: $729-475$.

68. Sasaki M, Nishida N, Shimada M (2018) Beneficial Role of Rooibos in Diabetes Mellitus: A Systematic Review and Meta-Analysis. Molecules 23: 839

69. Muller CJF, Malherbe CJ, Chellan N, Yagasaki K, Miura Y, et al. (2018) Potential of Rooibos, Its Major C-glucosyl Flavonoids, and Z-2-( $\beta$ D-glucopyranosyloxy)-3-phenylpropenoic Acid in Prevention of Metabolic Syndrome. Crit Rev Food Sci Nutr 58: 227-246.

70. Piek H, Venter I, Rautenbach F, Marnewick JL (2019) Rooibos Herbal Tea: An Optimal Cup and Its Consumers. Health SA 24: 1090.

71. Yang S, Lee C, Lee BS, Park EK, Kim KM, et al. (2018) Renal Protective Effects of Aspalathin and Nothofagin From Rooibos (Aspalathus linearis) in a Mouse Model of Sepsis. Pharmacol Rep 70: 1195-1201.

72. Johnson R, Shabalala S, Louw J, Kappo AP, Muller CJF (2017) Aspalathin Reverts Doxorubicin-Induced Cardiotoxicity through Increased Autophagy and Decreased Expression of p53/mTOR/p62 Signaling. Molecules 22: 1589

73. Johnson R, Dludla PV, Muller CJ, Huisamen B, Essop MF, et al. (2017) The Transcription Profile Unveils the Cardioprotective Effect of Aspalathin against Lipid Toxicity in an In Vitro H9c2 Model. Molecules 22: 219

74. Johnson R, Dludla P, Joubert E, February F, Mazibuko S, et al. (2016) Aspalathin, a Dihydrochalcone C-glucoside, Protects H9c2 Cardiomyocytes Against High Glucose Induced Shifts in Substrate Preference and Apoptosis. Mol Nutr Food Res 60: 922-934.

75. Schloms L, Smith C, Storbeck KH, Marnewick JL, Swart P, et al. (2014) Rooibos Influences Glucocorticoid Levels and Steroid Ratios in Vivo and in Vitro: A Natural Approach in the Management of Stress and Metabolic Disorders? Mol Nutr Food Res 58: 537-549.

76. Mazibuko SE, Muller CJ, Joubert E, de Beer D, Johnson R et al. (2013) Amelioration of Palmitate-Induced Insulin Resistance in $\mathrm{C}_{2} \mathrm{C}_{12}$ Muscle Cells by Rooibos (Aspalathus linearis). Phytomedicine 20: 813-819.

77. Pantsi WG, Marnewick JL, Esterhuyse AJ, Rautenbach F, van Rooyen J (2011) Rooibos (Aspalathus linearis) Offers Cardiac Protection Against Ischaemia/Reperfusion in the Isolated Perfused Rat Heart. Phytomedicine 18: 1220-1238.

78. van der Merwe JD, Joubert E, Richards ES, Manley M, Snijman PW, et al. (2006) A Comparative Study on the Antimutagenic Properties of Aqueous Extracts of Aspalathus linearis (Rooibos), Different Cyclopia Spp. (Honeybush) and Camellia sinensis Teas. Mutat Res 611: 42-53.

79. Lee EJ, Jang HD (2004) Antioxidant Activity and Protective Effect on DNA Strand Scission of Rooibos Tea (Aspalathus linearis). Biofactors 21: 285-292. 